\title{
Severe Astigmatic Blur Does Not Interfere with Spectacle Lens Compensation
}

\author{
Rbondalyn C. McLean and Josh Wallman
}

Purpose. Whether either natural emmetropization or compensation for imposed spectacle lenses requires the visual system to distinguish myopic from hyperopic blur is controversial. Some have argued that the visual system need only respond to the magnitude of the blur. This study was undertaken to test whether adding large amounts of astigmatic image blur would cause myopia and interfere with compensation for positive or negative spectacle lenses.

Methods. Chicks were fitted with mixed astigmatic (toric) lenses with $+5 \mathrm{D}$ on one meridian and $-5 \mathrm{D}$ on the orthogonal meridian (Jackson crossed cylinders), thus producing massive blur while having no spherical equivalent power. Chicks wore these lenses either alone or in combination with $+6 \mathrm{D},+3 \mathrm{D}$, $-3 \mathrm{D}$, or $-6 \mathrm{D}$ spherical lenses. To produce a similar degree of image degradation in a different way, other chicks were fitted with weak diffusers, with or without spherical lenses.

REsults. Eyes fitted with astigmatic lenses alone became mildly hyperopic, whereas those fitted with weak diffusers became mildly myopic. Eyes wearing both the astigmatic and spherical lenses compensated as completely for the spherical lenses as did the eyes wearing spherical lenses alone-the compensation being caused by changes in both choroidal thickness and ocular length. Eyes wearing weak diffusers and spherical lenses also compensated but did so without a change in choroidal thickness.

Conclusions. Severe astigmatic blur does not interfere with spectacle lens compensation. From this it can be inferred that the average amount of blur is not an important error signal for spectacle lens compensation and therefore probably not for emmetropization either. These results strengthen the case that the sign of defocus is used to direct eye growth. (Invest Ophthalmol Vis Sci. 2003;44:449-457) DOI:10.1167/iovs.010670

$\mathrm{T}$ he eyes of neonates of all species studied have rather variable refractive errors, generally hyperopic, that move toward emmetropia in the postnatal period. Although the eye could achieve emmetropia by autonomously growing toward the adult eye shape, with the curvature of the optical components changing to yield a focal length matched to the physical

From the Department of Biology, City College, City University of New York, New York, New York.

Supported by National Institutes of Health Grants EY02727 and RR-03060

Submitted for publication July 6, 2001; revised July 9, 2002; accepted July 31, 2002.

Commercial relationships policy: $\mathrm{N}$.

The publication costs of this article were defrayed in part by page charge payment. This article must therefore be marked "advertisement" in accordance with 18 U.S.C. $\$ 1734$ solely to indicate this fact.

Corresponding author: Josh Wallman, Department of Biology, City College, City University of New York, New York, NY 10031;

wallman@sci.ccny.cuny.edu. length of the eye, it is clear that the growth of the eye can also be guided by visual feedback. If the eyes of chicks, monkeys, or tree shrews have either myopia or hyperopia imposed on them by spectacle lenses, the eyes compensate for the imposed defocus to become functionally emmetropic. ${ }^{1-5}$ Two anatomic components change to effect this compensation: When hyperopia (image focused behind the retina) is imposed, the rate of ocular elongation increases and the choroid thins, thereby pulling the retina back toward the image plane. Conversely, when myopia is imposed, the rate of ocular elongation decreases and the choroid thickens, pushing the retina forward toward the image plane.$^{6,7}$ Because the eye is able to compensate for both negative and positive lenses, this could be regarded this as prima facie evidence that the eye is able to distinguish between hyperopic and myopic blur and to respond with growth in either direction.

However, it can also be argued that the eye need not distinguish the sign of its refractive error to accomplish compensatory growth responses to positive and negative spectacle lenses. Instead, these could occur as a consequence of the eye's growing in the direction of myopia if vision were blurred and growing in the direction of hyperopia if vision were sharp. Put differently, the amount of ocular elongation may simply reflect the average amount of blur or image degradation: If the eye sees more blur, the eye elongates more; if the eye sees less blur, it elongates less. ${ }^{8}$ This argument, which we refer to as the quantity-of-blur hypothesis depends on two premises: that young animals generally view nearby objects and that accommodation is imperfect, so that some blur remains. As a consequence, even emmetropic eyes would experience some blur, which would contribute to maintaining a normal rate of ocular elongation. Eyes with hyperopia (including that imposed by the wearing of negative lenses) would experience even more blur, which would speed ocular elongation even more, resulting in compensation for the hyperopia. Only eyes with myopia (including that imposed by positive lenses) would see sharp images, and this would halt elongation, resulting in growth toward hyperopia as the focal length of the eye continued to increase with age. Such a control mechanism would maintain emmetropia by negative feedback because errors in the direction of hyperopia would increase blur and hence elongation, thereby reducing the hyperopia, whereas errors in the direction of myopia would decrease blur and elongation, thereby reducing the myopia, thus in both cases tending to drive the eye back toward emmetropia.

If this quantity-of-blur hypothesis is correct, any device that increases blur, even without creating a net spherical refractive error, would have several consequences. First, it would drive an emmetropic eye toward myopia. Second, it would increase the response to negative spectacle lenses by adding to the blur-driven compensatory ocular elongation. Third, it would reduce the effect of positive spectacle lenses by reducing the sharp vision, thus reducing the predicted slowing of ocular elongation that would lead to compensatory hyperopia. We tested whether the average amount of blur guides eye growth, 


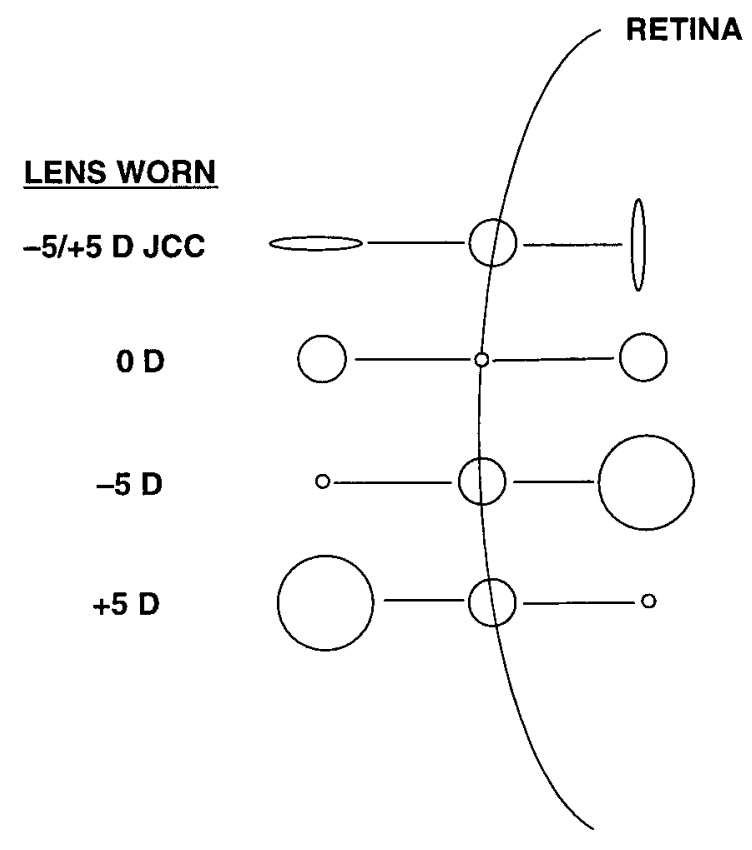

FiguRE 1. The effect of spherical lenses and of +5/-5-D crossed astigmatic lenses (Jackson crossed cylinders, JCC) on the blur circle of a point source imaged by an emmetropic eye. If no spectacle lens is worn $(0 \mathrm{D})$, the image is focused at the retina, and the size of the blur circle increases both in front of and behind the retina. Positive and negative spherical lenses focus the image in front of or behind the retina, respectively, with increasing blur circles away from the plane of focus. The astigmatic lenses used in this study had no spherical power, but the two axes focused images $10 \mathrm{D}$ apart (the interval of Sturm). Within this interval, moving toward or away from the retina reduces the blur for one axis and increases it for the other axis. In the middle of this interval (at the retina in this diagram), the blur circle is round, but much larger than in the case of the emmetropic eye without lenses.

by raising chicks with mixed astigmatic (toric) lenses without spherical equivalent power (Jackson crossed-cylinder lenses, $+5 \mathrm{D}$ along one axis, $-5 \mathrm{D}$ along the orthogonal axis; called here, +5/-5-D lenses or simply astigmatic lenses). By having chicks wear these lenses, we tested whether the greatly increased blur would drive the eye toward myopia. By having them worn together with spherical lenses, we tested whether the additional average blur would augment the effect of negative lenses and subtract from the effect of positive lenses. For comparison, to increase the overall level of image degradation in a manner different from the astigmatic lenses, we superimposed weak diffusers on the spectacle lenses. Unlike strong diffusers, which prevent form vision and therefore result in form-deprivation myopia, weak diffusers permit impaired form vision and cause only mild myopia in both chicks and monkeys. 9,10

To clarify the way in which spherical lenses and our astigmatic lenses add, Figure 1 shows how several lenses affect the size of the blur circle of a point source at different image planes. Note that the crossed astigmatic lenses that we used (Jackson crossed cylinders), although they have no spherical power, cause the blur circle to be the same size on the retina as in an eye wearing a +5-D or a -5-D lens. This arises because, in one meridian, the crossed astigmatic lens would focus light exactly like a +5-D lens, whereas in the orthogonal meridian it would focus light like a -5 -D lens. ${ }^{11,12}$ As the image plane is moved either in front of or behind the retina (by changing the state of accommodation or by adding a spherical lens), the blur circle of a point source in an eye wearing the astigmatic lens becomes an increasingly elongated ellipse until one orientation is correctly focused, whereas the orthogonal orientation is 10 D out of focus-all other orientations being substantially blurred in any plane. As a consequence, if the image of an object were moved back and forth within this interval (the interval of Sturm), the image would not be brought in and out of focus, but instead the focus along one astigmatic meridian would improve at the expense of the quality of focus along the orthogonal meridian. Therefore, the average quality of focus (or the average blur) would change much less than normal as the object was moved back and forth. One could say that the depth of focus of the eye has been greatly increased by adding the astigmatic lens, because it now requires a larger defocus to cause a detectable change in the average defocus.

This experiment also provided the opportunity to explore the possibility that the eye's inherent astigmatism may serve as an error signal guiding emmetropization. For example, in an eye with no spherical error and with-the-rule (WTR) astigmatism (more power vertically than horizontally), the blur circle of a point source would become more vertically elongated if the eye grew in the myopic direction and more horizontally elongated if the eye grew in the hyperopic direction. Support for the possibility that astigmatism is used this way comes from an abstract asserting that wearing simple astigmatic lenses (lenses with optical power in only one axis) results in shifts in spherical refractive error that were dependent on the orientation of the lens, ${ }^{13}$ as well as the finding in children that eyes with against-the-rule (ATR) astigmatism are predisposed to become myopic. ${ }^{14}$ We looked for differences in refractive error resulting from differences in the orientation of the imposed astigmatic lenses.

Finally, this experiment also permitted us to test the claim that the chick eye can compensate for imposed astigmatism. ${ }^{2,15}$ We investigated whether eyes wearing our +5/-5-D astigmatic lenses would develop a compensatory astigmatism dependent on the lens orientation.

\section{MeTHODS}

\section{Animals}

White Leghorn chickens (Gallus gallus domesticus, Cornell K-strain) obtained from Cornell University (Ithaca, NY) 1 day after hatching, were raised in a 14-10-hour light-dark cycle. During experiments, chickens were kept in a cage of $0.5 \mathrm{~m}^{2}$ inside a closed, heated, sound-attenuated chamber. Lenses were inspected twice daily and cleaned as needed. Use of animals conformed to the ARVO Statement for the Use of Animals in Ophthalmic and Vision Research.

\section{Lenses}

Lenses were attached to rigid plastic rings mounted on Velcro support rings. Mated Velcro rings were attached with collodion to the feathers around one eye of each bird. Astigmatic lenses with $+5 \mathrm{D}$ along one axis and $-5 \mathrm{D}$ along the orthogonal axis, $12 \mathrm{~mm}$ in diameter, were made from CR39 plastic (Tri-Supreme Optical, Farmingdale, NY). Spherical lenses (plano [no optical power], $+3 \mathrm{D},+6 \mathrm{D},-3 \mathrm{D}$, and -6 D) were made from PMMA with a base curve of $7 \mathrm{~mm}$ and an optic zone diameter of $10 \mathrm{~mm}$ (Conforma Contact Lens Co., Norfolk, VA). To combine astigmatic lenses with spherical ones, the spherical lenses were placed on top of the astigmatic lenses, and both were fitted into a clear plastic tube. The resultant lenses had the following powers along their two orthogonal axes: $+8 /-2 \mathrm{D},+11 /+1 \mathrm{D},+2 /-8 \mathrm{D}$, and $-1 /-11 \mathrm{D}$, respectively.

Lenses were fitted over one eye at 6 days of age. To align the lenses in relation to the normal head angle, chicks were photographed from the side in their cages and an estimate made of the average angle between the horizontal and the line formed by the auditory meatus and the beak tip-an angle of $18^{\circ}$ (beak down). Consequently, we marked the Velcro rings that the birds wore at that angle, and we fitted the 

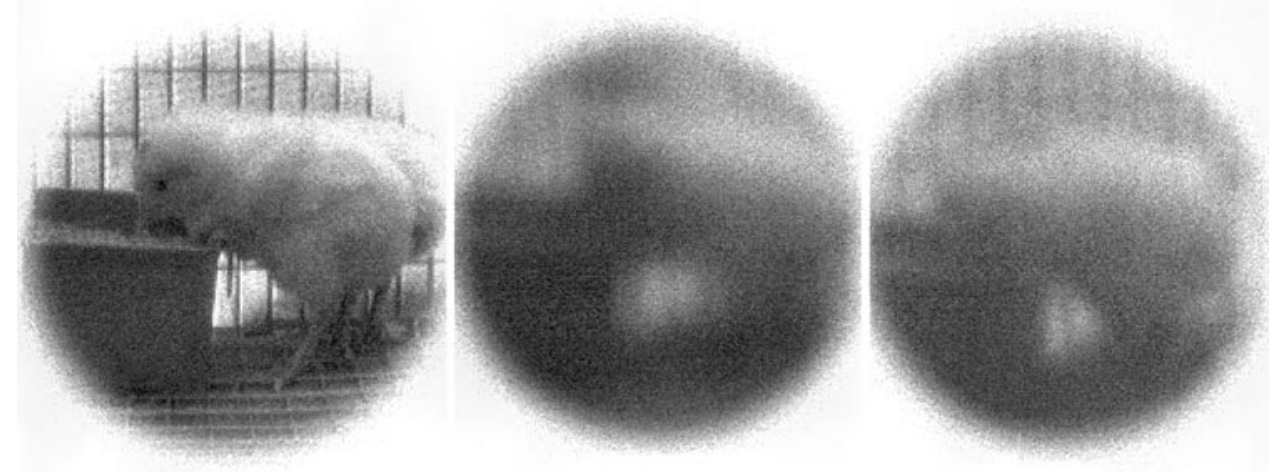

FIGURE 2. Simulation of the optical effect of astigmatic lenses and diffusers. Photographs were taken with a video camera with a focal length similar to that of the chick eye $(8 \mathrm{~mm})$ and a pupil diameter slightly larger than that of the chick eye $(4 \mathrm{~mm}$ vs. $3 \mathrm{~mm}$ ). Because the mixed astigmatic lens had no spherical power, we did not refocus the camera after adding this lens. Had we refocused the camera to bring one or the other of the axes of the astigmatic lens into focus, the astigmatism would have been much more evident: photograph through normal lens (left), photograph through astigmatic lens (middle), photograph through the denser of the two diffusers (right).

astigmatic lenses either in the WTR or ATR orientation or at $45^{\circ}$. When combined with spherical lenses, the astigmatic lenses were always oriented at $45^{\circ}$. This orientation produced maximum image distortion, because it blurred the vertical and horizontal bars of the chicks' cage. It also caused maximum image motion during horizontal or vertical head movements.

\section{Diffusers}

To obtain different degrees in reduction of image sharpness, Bangerter occlusion foils (Ryser Optik, St. Gallen, Switzerland) were used. These lightly frosted flexible diffusers are manufactured for use with human spectacle lenses. The diffusers were cut into 8-mm circles and applied wet to the posterior surface of the lenses worn by the chicks. Because of the steep curvature of the lenses, it was not possible to cover the inside of the lens completely with the diffusers, and so a white cloth ring covered the gap between the diffuser and the outer rim of the lens. This reduction in aperture size has been reported not to impair lens compensation. ${ }^{15}$ Diffusers of two different degrees ( 0.4 [more dense] and 0.8 [less dense]) were placed inside plano, +6-D, and -6-D lenses. As a control, +6-D, and -6-D lenses were used alone.

\section{Optical Effects of Astigmatic Lenses and Diffusers}

To characterize very approximately the optical effect on the chicken eye of the astigmatic lenses and diffusers, we photographed a scene at $25 \mathrm{~cm}$ with a video camera somewhat similar to the chick eye (focal length, $8 \mathrm{~mm}$; pupil diameter, $4 \mathrm{~mm}$ ). The resultant images (Fig. 2) were transformed into the spatial frequency domain and the power spectra were summed radially (Fig. 3, calculations by NIH Image program; produced by W. Rasband, National Institutes of Health; available by ftp from zippy.nimh.nih.gov). This computation confirms the visual impression that the astigmatic lenses degrade the image more than the diffusers do. The effects of these diffusers on the human contrast sensitivity function has been described. ${ }^{16}$

\section{Measurements}

Both before lens fitting and after 2 to 6 days of lens wear, refractive error measurements were made using a Hartinger refractometer (Zeiss, Jena, Germany), modified for small pupils. Chicks were measured while under halothane anesthesia (1.5\% in oxygen) without cycloplegic agents, although we find that halothane produces a moderate degree of mydriasis and presumably of cycloplegia as well. The lids were retracted with extremely light retractors made from spring wire. Care was taken in the placement of the retractors to minimize any pressure on the globe. The axis of the refractometer was aligned with the pupillary axis of the eye by rotating the head of the bird to center in the pupil a ring-light coaxial with the optical axis of the instrument, with the refractometer used as a telescope. Once aligned, the refractometer was translated forward toward the eye to make the measurements. After several measurements, the instrument was realigned with the eye and a new set of measurements made.

A-scan ultrasonography with a 30-MHz polymer transducer sampled at $100 \mathrm{MHz}$ was used to measure internal axial dimensions (for detailed methods, see Ref. 17). We calculated the thickness of each axial

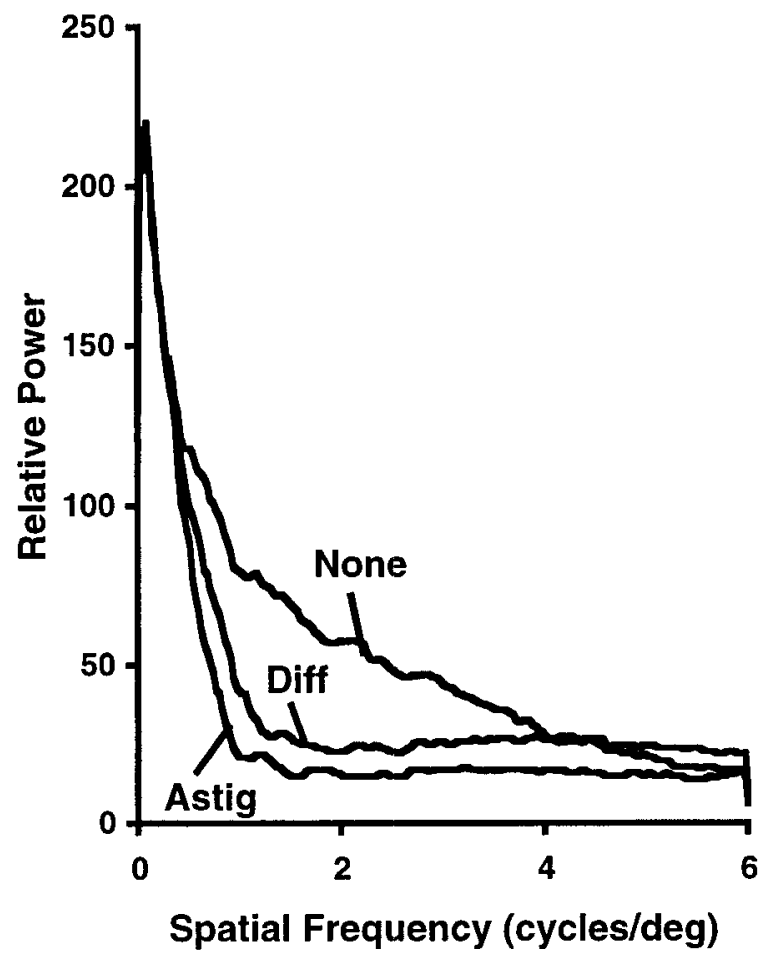

Figure 3. Power spectra of images in Figure 2. Images were masked, a fast Fourier transform performed on computer, and the power spectra averaged across orientations. The mixed astigmatic lenses (Astig) had as much or more effect on the power spectrum as did diffusers (Diff), in comparison with the photograph through the normal lens (None). Powers are relative values (normalized for each curve) on an arbitrary logarithmic scale. 


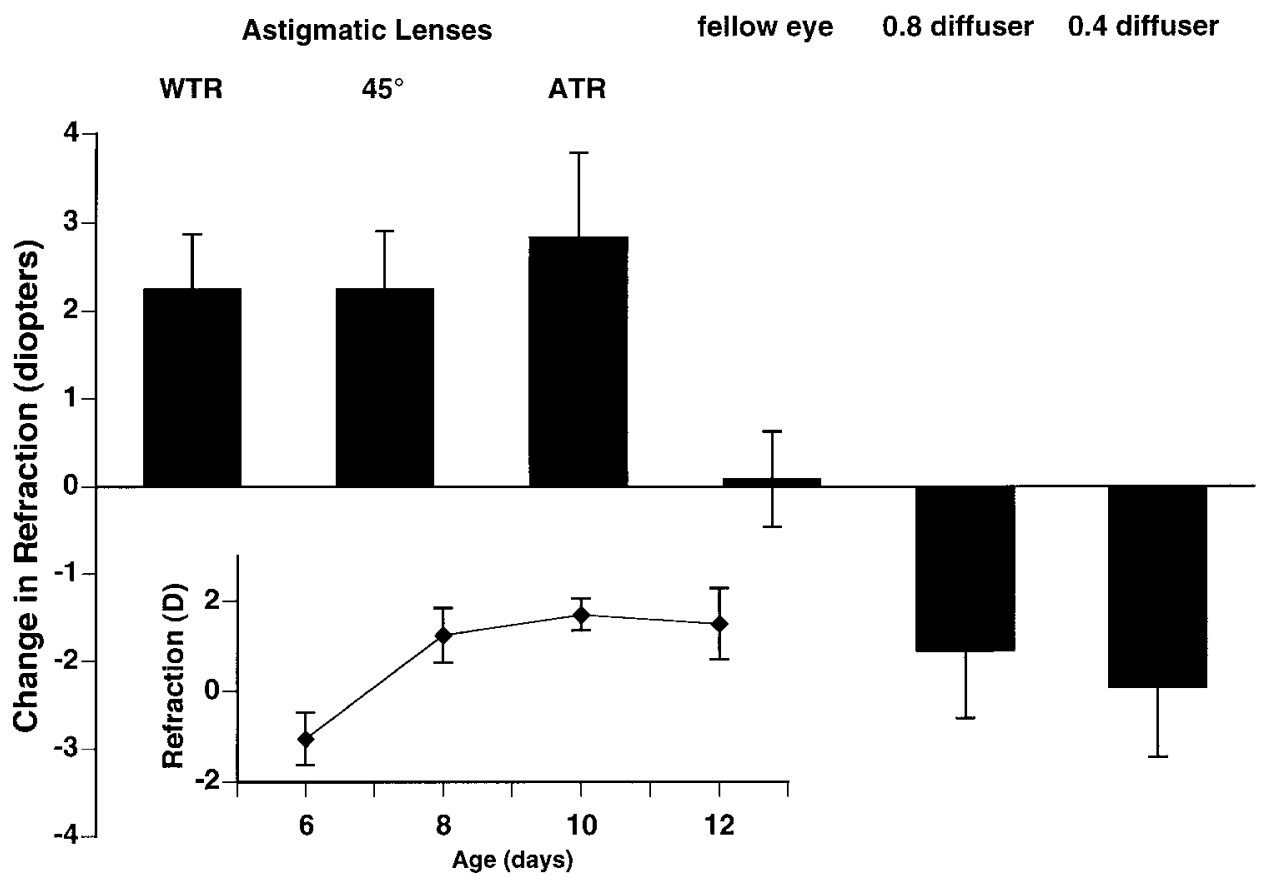

Figure 4. Changes over 2 days of wearing either +5/-5-D astigmatic lenses oriented at different angles (ATR, $n=7$; WTR, $n=9 ; 45^{\circ}, n=$ 8) or weak diffusers (0.8 diffuser, $n$ $=12 ; 0.4$ diffuser, $n=9$ ). Inset: time course of refractive change in eyes wearing astigmatic lenses oriented at $45^{\circ}(n=8$, except $n=4$ at 12 days). structure from the cornea to the external scleral surface, using the appropriate velocity of sound for each structure. ${ }^{18}$ To obtain the ocular length, we summed all these distances. This measure differs from the common clinical convention of regarding axial length as the distance from cornea to retina. Because our ocular length is the length of the eye globe, it would not be affected by expansion of the choroid, which would reduce the conventional (clinical) axial length, just as it would reduce the vitreous chamber depth. Thus, we have independent measures of the two principal components of lens compensation, length of the eye and thickness of the choroid.

\section{RESUlts}

\section{Astigmatic Lenses Alone Versus Diffusers Alone}

Wearing astigmatic lenses without spherical power did not cause the eyes to shift toward myopia. Instead, there was a significant shift in the opposite direction (Fig. 4; mean change over 2 days: experimental eye, $+2.5 \mathrm{D}$, fellow eye, $+0.1 \mathrm{D}$; paired $t$-test across all subgroups, $P<0.01$ ). Twenty-four of 29 eyes changed in the direction of hyperopia. This mild hyperopia lasted for at least 6 days (Fig. 4, inset). There was no significant difference in refraction among the three orientations at which the lenses were worn (ANOVA, $\mathrm{F}=1.82, P>$ 0.05). Ultrasonography showed that this hyperopic shift was due to inhibition of the normal axial elongation. The mean change in ocular length over 2 days was only $6 \pm 50 \mu \mathrm{m}$ (SD) in the experimental eyes, compared with $150 \pm 70 \mu \mathrm{m}$ in the untreated fellow eyes, a significant difference (paired $t$-test $P<$ 0.0001 , lenses fitted WTR and ATR combined; $n=21$ ). The choroid thickness was not affected (change over 2 days in experimental eyes, $21 \pm 70 \mu \mathrm{m}$; untreated fellow eyes, $13 \pm$ $40 \mu \mathrm{m}, P>0.05$ by paired $t$-test, $n=21$ ).

In contrast, weak diffusers, which degraded the image less than the astigmatic lenses, resulted in a myopic shift (Fig. 4; mean change for plano lenses with 0.8 diffuser $=-1.9 \mathrm{D}$, for 0.4 diffuser $=-2.3 \mathrm{D}$ ) and a normal rate of ocular elongation (307 $\pm 117 \mu \mathrm{m}, n=15$ ) over 4 days, in marked contrast to the inhibition of elongation in eyes wearing astigmatic lenses.

\section{Lens Compensation: Astigmatic Lenses Versus Diffusers}

When the astigmatic lenses were combined with spherical lenses, the eyes compensated for the spherical error imposed as though the astigmatic lenses were not present (Fig. 5). The change in the eye's refractive error with either the $+6-\mathrm{D}$ or -6-D lenses was not significantly affected by the presence of the astigmatic lens (Fig. 5A; change over 2 days: +6-D lens alone was $+5.0 \mathrm{D}$ vs. +6-D lens with astigmatic lens, which was +4.9 D; -6-D lens alone was $-2.1 \mathrm{D}$ vs. -6 -D lens with astigmatic lens, which was $-3.7 \mathrm{D}$; in both cases $P>0.05$ by $t$-test; $n=7$ to 12 ).

Even lenses of lower spherical power were compensated for. When the astigmatic lens was combined with the $-3-\mathrm{D}$ lens, the mean refractive error changed by $-2.5 \pm 1.9 \mathrm{D}$ over 2 days, compared with a change of $+2.7 \pm 1.3 \mathrm{D}$ for $+3-\mathrm{D}$ lenses, a significant difference $(P<0.0001$, $t$-test, $n=7$ in each group, Fig. 5B). Of these two lens conditions, only the difference between the astigmatic lens with the -3-D lens was significantly different from the astigmatic lens alone. The compensation for these weak lenses is more surprising than that for \pm 6 -D lenses, because in this case, the quantity of blur imposed by the spherical lenses was a minor component of the total blur. To be specific, as shown in two recent reports, ${ }^{11,12}$ the total blur is best represented by the resultant vector of the three orthogonal refractive components (the spherical equivalent and the two Fourier components of the astigmatism). In the present case, the astigmatic lens contributes $5 \mathrm{D}$ of the total blur of $5.8 \mathrm{D}$.

The changes in the ocular components responsible for the refractive compensation were similar, whether or not astigmatic lenses were worn. In both cases, the change in refractive error was largely axial, as shown by the slope of $20 \mathrm{D} / \mathrm{mm}$ of the regression line between refraction and vitreous chamber depth, close to the $17 \mathrm{D} / \mathrm{mm}$ that would be predicted if only the axial length changed in birds of this age (for individual eyes, slope $=22 \mathrm{D} / \mathrm{mm}$ for spherical lenses with astigmatic lenses; $17 \mathrm{D} / \mathrm{mm}$ for spherical lenses alone; slopes not significantly different, $t=1.1, d f=20, P>0.05$; for group data, see Fig. 6A). Furthermore, in both cases the compensation for 

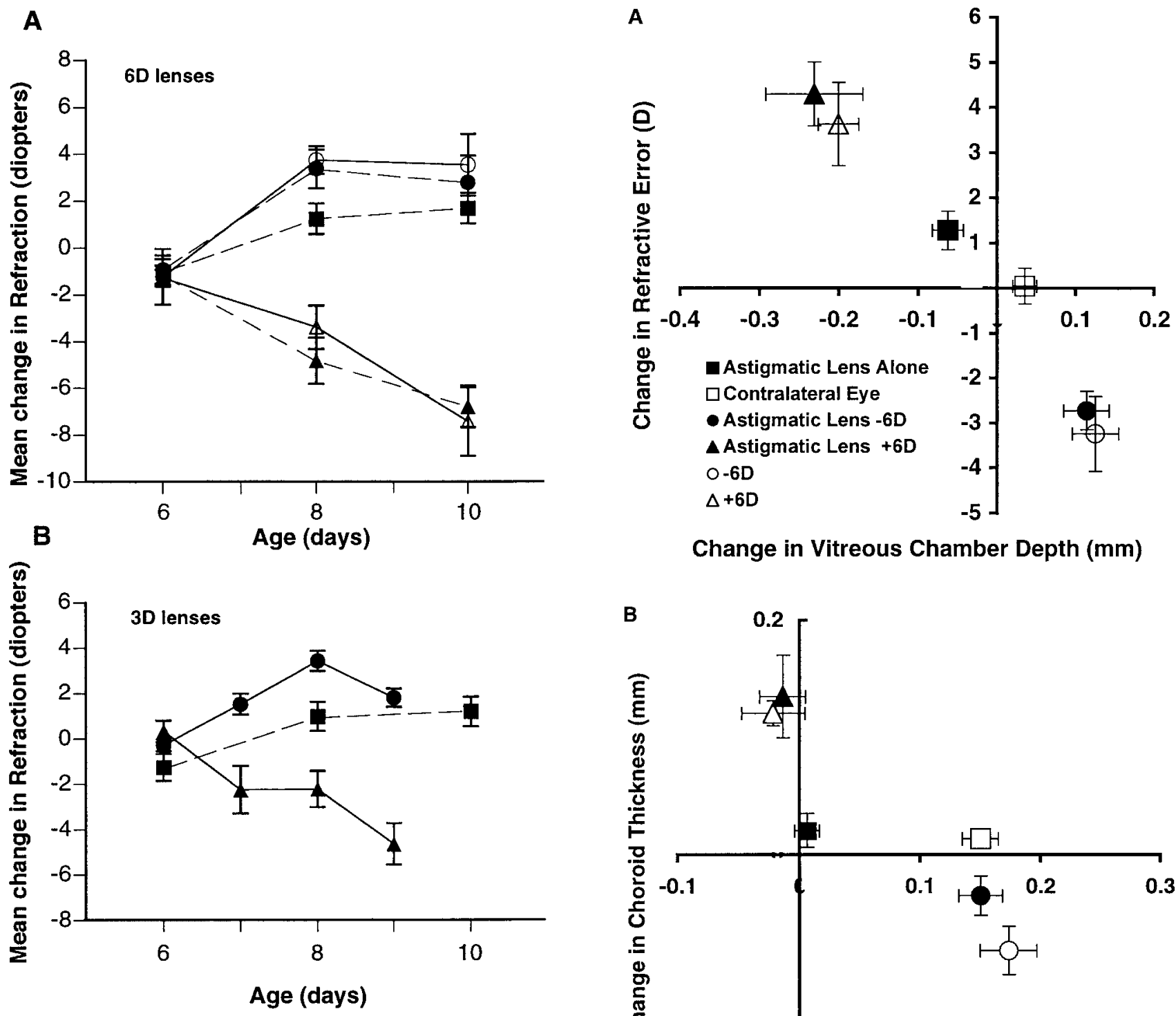

\section{Change in Vitreous Chamber Depth ( $\mathrm{mm})$}

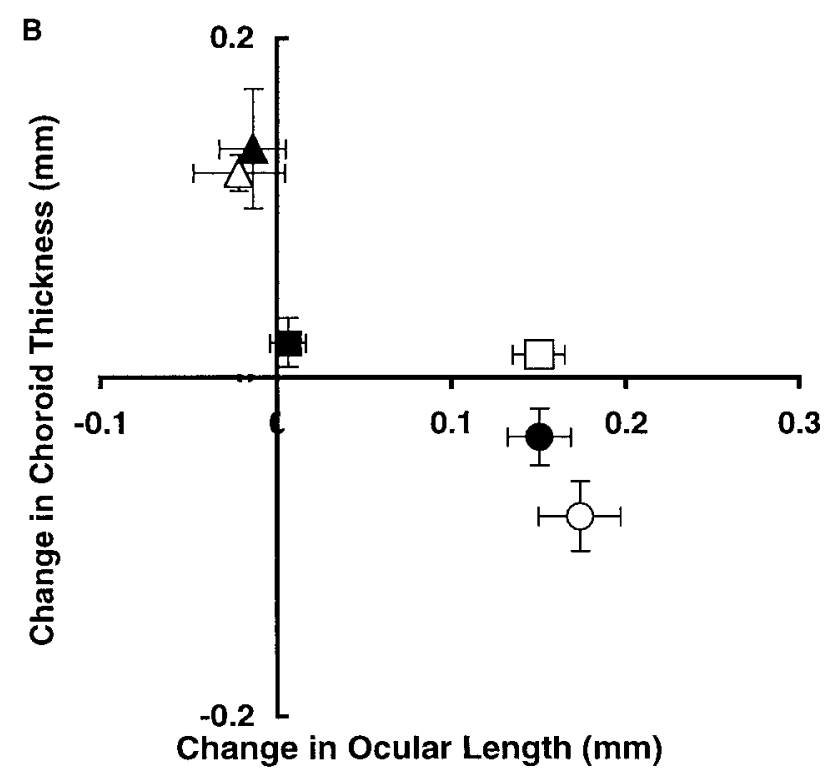

FIGURE 6. Summary of data for groups of chicks wearing spherical lenses alone or in combination with astigmatic lenses. (A) The spherical lenses had similar effects on both refractive error and vitreous chamber depth, whether or not in they were worn in combination with the astigmatic lenses. The eyes wearing astigmatic lenses alone were more hyperopic and had shorter vitreous chambers than the untreated fellow eyes. (B) The spectacle lens compensation was associated with similar changes in choroidal thickness and ocular elongation, whether or not the spherical lenses were worn in combination with the astigmatic lenses. The vertical error bars of the contralateral eye do not appear, because they are smaller than the symbol. $(n=6$ for each group, except for eyes wearing astigmatic lenses alone and for their untreated fellow eyes, for which $n=16$ in (A) and $n=22$ in (B). The eyes represented are the WTR and ATR eyes in Figure 4, except for five eyes omitted because ultrasound measurements were not available.

negative lenses was accomplished principally by increased ocular elongation and secondarily by thinning of the choroid, and the compensation for positive lenses was accomplished by slowed ocular elongation and thickening of the choroid (Fig. 6B). The eyes wearing astigmatic lenses alone showed an

inhibition of ocular length without a change in choroidal thickness.

These results showing normal compensation for spectacle lenses with astigmatic lenses indicate that sharp images are not 


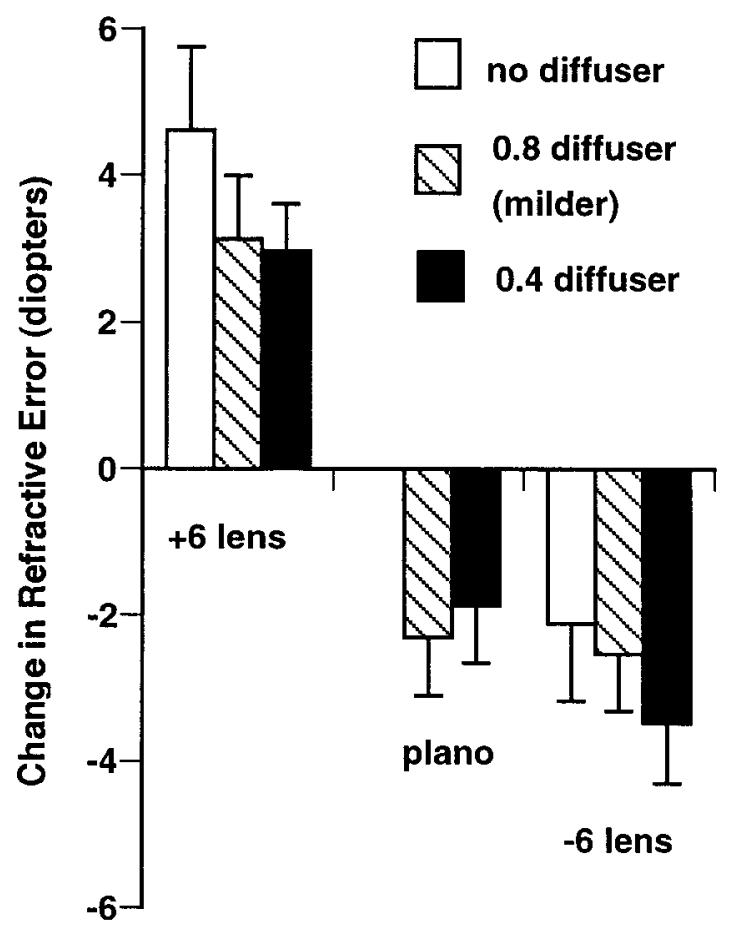

FIGURE 7. Changes in refractive error over 2 days in eyes wearing spherical lenses in combination with weak diffusers.

necessary and that image degradation does not necessarily result in form-deprivation myopia.

The diffuser experiments, as well, indicate that sharp images are not required for spectacle lens compensation. The refractive compensation for spherical lenses worn together with either type of diffuser was similar to that with spherical lenses alone (Fig. 7). Eyes wearing +6-D lenses with diffusers changed in the direction of hyperopia (mean change over 2 days with 0.8 diffuser $=+3.1 \mathrm{D}$ and with 0.4 diffuser $=+3.0$ $\mathrm{D}$; in both cases $P<0.05$ by $t$-test; $n=7-9$ ). Eyes wearing -6 -D lenses with diffusers induced eye growth in the myopic direction (mean change over 2 days with 0.8 diffuser $=-2.5$ $\mathrm{D}$ and with 0.4 diffuser $=-3.5 \mathrm{D}$; in both cases $P<0.01$ by $t$-test; $n=8$-10). Thus, there was statistically significant compensation for both $+6-\mathrm{D}$ and -6 -D lenses, despite the diffusers. Furthermore, there was no statistically significant effect of wearing either type of diffuser on the compensation for either the $+6-\mathrm{D}$ or -6 -D lenses ( $P>0.05$ for all groups, $n=7-10$ ), although there was a hint that the eyes wearing diffusers with lenses were slightly more myopic than those wearing the lenses alone, suggesting that the effects of the diffusers and the lenses are additive, but not significantly so.

The ocular component changes accompanying lens compensation in the presence of the diffusers were clearly axial, as was the case with the spherical lenses alone and the spherical lenses plus astigmatic lenses. The slope of the regression line of change in refractive error on change in vitreous chamber depth was exactly the same $(15.4 \mathrm{D} / \mathrm{mm})$ with the two densities of diffusers, but offset $1 \mathrm{D}$ toward more myopic refractions in the case of the more dense diffusers. However, in marked contrast to the component changes observed in the compensation for the spherical lenses alone or in combination with the astigmatic lenses, the diffusers prevented the large choroidal differences normally observed between the $+6-\mathrm{D}$ and $-6-\mathrm{D}$ lenses (the choroids of the eyes wearing lenses and diffusers differed in thickness by only $8 \mu \mathrm{m}$; eyes wearing lenses without diffusers differed by $170 \mu \mathrm{m})$. Instead, all the compensation was accomplished by changes in ocular length (mean =
$+59 \pm 79 \mu \mathrm{m}$, for the +6 -D lenses with diffusers, and $+388 \pm$ $125 \mu \mathrm{m}$, for the $-6-\mathrm{D}$ lenses with diffusers). This suggests that the visual requirements for changes in choroidal thickness differ markedly from those for the changes in the rate of ocular elongation.

\section{Other Effects of Imposed Astigmatism}

If eyes used their intrinsic astigmatism to guide emmetropization, it would be expected that adding to or reversing this astigmatism by our imposed astigmatic lenses might cause the eye to change its refractive error in a direction that depended on the orientation of the astigmatic lens and the eye's initial axis of astigmatism. Contrary to a published abstract, ${ }^{13}$ our results show no difference in the effect of our astigmatic lenses on spherical equivalent refraction related to the orientation of the lens (Fig. 4; ANOVA, $\mathrm{F}=1.82, P>0.05$ ). Even restricting our analysis to 19 eyes showing WTR astigmatism, we found no evidence that the axis of astigmatism guides change in refractive status. Specifically, those wearing lenses in the WTR orientation changed by $+2.8 \pm 1.9 \mathrm{D}, n=9$ ) over 2 days, and those wearing lenses in the ATR orientation changed by $+2.2 \pm 3.5 \mathrm{D}, n=10$ ).

Furthermore, we did not find that eyes compensated for the imposed astigmatism. To show the changes in both the spherical refraction and astigmatism when the astigmatic lenses alone were worn, we present the refractions in relation to the first two terms of the Fourier decomposition (Fig. 8). Clearly, most of the arrows are more horizontal than vertical, indicating that most eyes made larger spherical than astigmatic refractive changes. We found no significant differences between the eyes wearing astigmatic lenses in the WTR and ATR configurations, although there was a nonsignificant tendency for the eyes wearing astigmatic lenses in the WTR orientation to change more in spherical equivalent and in the $J_{0}$ (cosine) component $(P>0.05, t$-test, Fig. 8, inset).

\section{Discussion}

Our principal results are that the addition of large amounts of astigmatic blur did not shift the eyes toward myopia, but toward hyperopia, and did not interfere with either compensation for the hyperopic defocus imposed by negative spectacle lenses or compensation for the myopic defocus imposed by positive lenses. Weak diffusers, in contrast, shift the eye toward myopia, as also shown by Bartmann and Schaeffel ${ }^{9}$ in the chick and by Smith and Hung ${ }^{16}$ in the monkey. When added to the negative or positive lenses, weak diffusers do not significantly impair lens compensation, but the choroidal component of the compensation is much reduced.

\section{Other Studies}

Three other studies have tested chicks with either astigmatic blur alone, or with a combination of cylindrical and spherical blur. Thibos et al. ${ }^{19}$ found that chick eyes wearing $-10 \mathrm{D}$ cylinders with $+5 \mathrm{D}$ spheres (optically equivalent to our +5 / -5-D astigmatic lenses) become $2 \mathrm{D}$ more hyperopic than eyes wearing plano lenses (similar to the $+2.5 \mathrm{D}$ that we found). When both spherical and cylindrical blur are presented, Irving et al. ${ }^{15}$ found that cylindrical lenses causes compensation that approximated the spherical equivalent of the lenses (that is, +10 -D cylinders produce $+4.25 \mathrm{D}$ of compensation; -9-D cylinders produce $-3.5 \mathrm{D}$ of compensation), and Thibos et al. ${ }^{19}$ found that -10 -D astigmatic lenses result in refractions 5 D more myopic than those produced by astigmatic lenses with zero spherical power. All these results are similar to our results.

In contrast, Schmid and Wildsoet ${ }^{20}$ found that wearing simple cylindrical lenses causes nearly complete compensation 
FIGURE 8. Effect of wearing astigmatic lenses without spherical power on changes in spherical equivalent refraction (borizontal axis) and changes in astigmatism (vertical axis, cosine component of the Fourier expansion). Lenses were aligned in either the WTR (solid lines) or ATR (dashed lines) orientations. The foot of each arrow is the refraction before the lenses were fitted; the bead is the refraction after 2 days of lens wear. Eyes fitted with astigmatic lenses without spherical power did not compensate significantly for the astigmatic error imposed. Inset: mean vectors.

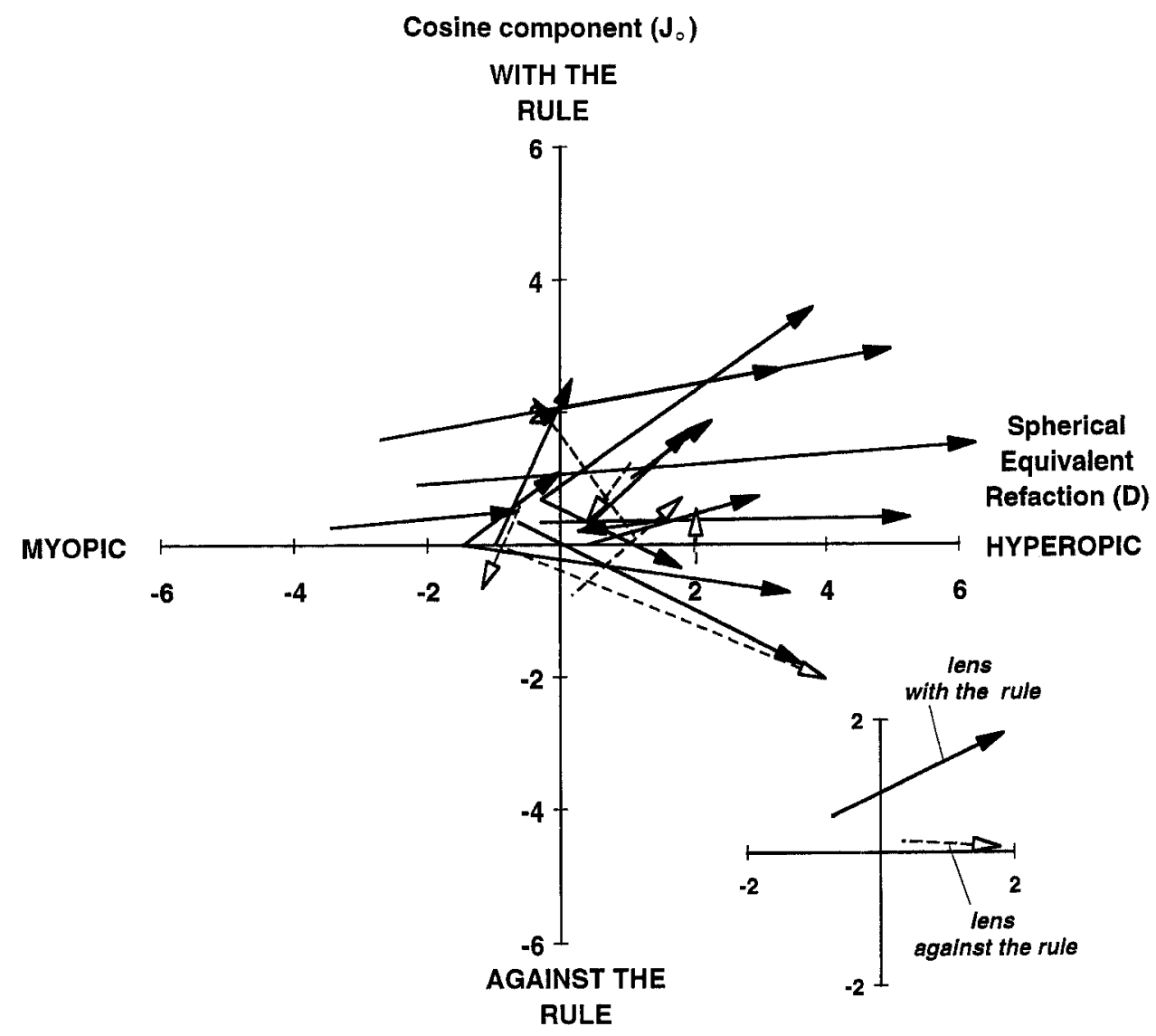

sharp images. Our results argue against this hypothesis on two counts: First, substantially degrading the image quality by the astigmatic lenses without spherical power caused a modest change toward hyperopia, whereas a similar degree of image degradation by a weak diffuser causes a change toward myopia. Second, eyes showed good compensation for the defocus imposed by spherical lenses, even in the face of much greater astigmatic blur. This point was shown most convincingly by the differential compensation shown for +3 - and -3-D lenses despite the presence of the astigmatic lenses. In these cases, the spherical lenses would account for only $18 \%$ of the $5.8 \mathrm{D}$ of blur produced by the combination of spherical and astigmatic lenses, as calculated by the method of Thibos et al. ${ }^{12}$ Nevertheless, compensation occurs, arguing that the magnitude of blur is not a parameter crucial to the compensation.

\section{Other Possible Cues}

If the quantity of blur is not the essential cue guiding lens compensation and, presumably emmetropization, what cues might be used? One cue that may survive the image degradation imposed by our crossed astigmatic lenses is the chromatic signal arising from the longitudinal chromatic aberration of the eye. Because short-wavelength light is refracted more strongly than long-wavelength light, myopically defocused objects would have the long-wavelength light sharply focused and the short-wavelength light blurred, whereas hyperopically defocused objects would have the reverse. Thus, the blur circle would be more red in the center and more blue on the outside for myopic defocus (that is, more of the blur circle would be blue than red) and the reverse for hyperopic defocus. With our astigmatic lenses, each blur circle would consist of some radial segments more red on the outside and others more blue on the outside. Averaging around the blur circle could give a veridical 
measure of whether the eye were myopically or hyperopically defocused, but only if the red and blue were averaged by area, not by intensity. By such a criterion, the blur circles lying on the retina in the schematic illustration in Figure 1 would be easily identifiable as emmetropic, myopic, or hyperopic, regardless of their size, by whether they were mostly red, mostly blue, or equally red and blue. Thus, the chromatic cue to defocus may not be substantially degraded by our astigmatic lenses.

Another possible cue may be that the combined effect of various monocular aberrations causes images blurred in the hyperopic direction to appear different from those blurred in the myopic direction. This manifests itself as a difference in the modulation transfer functions and, consequently, in the contrast sensitivity functions, between the two directions of defocus. ${ }^{24}$ Because these differences occur at relatively high spatial frequencies, this cue seems to be compromised by the large amount of blur our astigmatic lenses create.

Accommodation may also be used as a cue to the sign of defocus. Because hyperopic eyes would, on average, accommodate more than myopic eyes, if the brain monitored the average amount of accommodation, this would yield a measure of whether the defocus was myopic or hyperopic. If, as has been suggested, the eye were to accommodate to the meridian requiring the least accommodation, our crossed astigmatic lenses would reduce the accommodative demand and thus the amount of accommodation, thereby simulating myopia. Perhaps this accounts for the modest shift toward hyperopia that we observed when the astigmatic lenses were worn alone. However, we also expected that accommodation would be impaired by our astigmatic lenses, because changes in accommodative output would cause a much smaller change in the amount of defocus than would normally be the case, thus impairing the normal feedback that guides accommodation.

\section{Temporal Factors}

Whatever error signals are used in lens compensation, the effect of an astigmatic lens differs from that of a spherical lens or weak diffuser, in that contours aligned with one of the axes of the crossed astigmatic lens are occasionally seen in sharp focus. Thus, any process that makes use of these moments of relatively sharp vision may escape the image-degrading effects of the astigmatic lenses. Furthermore, the astigmatic lenses used in the current study would also have the effect of causing any small region of retina to experience alternating episodes of myopic and hyperopic defocus, depending on the orientation of the contours viewed. To estimate the effect of these fractionof-a-second episodes, we would have to know their temporal distribution. Recent work has shown that alternating the wearing of positive and negative lenses on a time scale of minutes causes compensation that greatly favors the effect of the positive lenses. ${ }^{25}$ Indeed, even a few minutes a day of positive lens wear can cancel the effects of wearing negative lenses the remainder of the day. ${ }^{26}$ However, as the frequency of alternation becomes faster (e.g., $2 \mathrm{~Hz}$ ), the net effect becomes closer to being purely additive, but still with a bias toward the positive lens. ${ }^{27}$ If the same asymmetry were true for alternations of myopic and hyperopic defocus imposed by the astigmatic lenses used in this study, this may explain the small hyperopic shift observed in eyes wearing the astigmatic lenses alone.

\section{Differential Effects of Diffusers and Astigmatic Lenses}

Our results that diffusers and astigmatic lenses have differential effects on choroidal thickness and ocular elongation raise the possibility that different retinal signals may control each. Specifically, we find that weak diffusers prevent the normal cho- roidal changes when spherical lenses are worn. In a related report, we show that when weak diffusers are added to positive lenses, they not only reduce the choroidal thickening, but enhance the inhibition of ocular elongation (Park T, Winawer $\mathrm{J}$, Wallman $\mathrm{J}$, manuscript submitted). In contrast, when astigmatic lenses are added to spherical ones, the refractive compensation involves normal changes in both choroidal thickness and ocular elongation, although the astigmatic lenses alone inhibit ocular elongation.

\section{Astigmatism as a Cue}

Beyond the effects on spectacle lens compensation, we also do not find that the axis of imposed astigmatism significantly influences spherical lens compensation, as might be expected to occur if the eye used its astigmatism as a cue to the sign of defocus. Furthermore, we find no evidence that the eye compensates for the imposed astigmatism. However, neither of these conclusions is definitive, both because we found hints of both effects and because it could be that the $10 \mathrm{D}$ of astigmatism imposed by our lenses was too great to be used as an error signal by the visual system. Thibos et al. ${ }^{19}$ and Schmid and Wildsoet $^{20}$ also failed to find astigmatic compensation; the latter investigators pointed out that those who had found such compensation $^{2,15}$ used a different strain of chickens (broilers). From our results we tentatively conclude that the eye's astigmatism is not used as a directional error signal in emmetropization and that imposed astigmatism is not eliminated through visual feedback. The second of these conclusions is not surprising, because lens compensation occurs primarily by changes in the back of the eye. Compensation for imposed astigmatism would require the cornea or lens to become more curved along one meridian.

\section{Conclusions}

In conclusion, we had chicks wear astigmatic lenses that present the retina with a view of the world that fluctuates in the degree and sign of refractive error but is on average much blurred. These lenses did not interfere with refractive compensation, leading us to conclude that lens compensation and probably emmetropization are not guided by the average absolute value of blur. Further work may reveal how the eye discerns the sign of defocus and compensates appropriately.

\section{Acknowledgments}

The authors thank Chea-Su Kee (University of Houston, Houston, TX) for a careful reading of an earlier draft and for alerting us to the conceptual confusion therein.

\section{References}

1. Schaeffel F, Glasser A, Howland HC. Accommodation, refractive error, and eye growth in chickens. Vision Res. 1988;28:639-657.

2. Irving EL, Sivak JG, Callender MG. Refractive plasticity of the developing chick eye. Opbthalmic Pbysiol Opt. 1992;12:448- 456.

3. Hung L-F, Crawford MLJ, Smith EL. Spectacle lenses alter eye growth and the refractive status of young monkeys. Nat Med. 1995; $1: 761-765$.

4. Smith E, Hung L. The role of optical defocus in regulating refractive development in infant monkeys. Vision Res. 1999;39:14151435 .

5. Siegwart JT, Norton TT. Refractive and ocular changes in tree shrews raised with plus or minus lenses [ARVO Abstract]. Invest Opbthalmol Vis Sci 1993;34(4):S1208. Abstract nr 2482.

6. Wallman J, Wildsoet $\mathrm{C}, \mathrm{Xu} \mathrm{A}$, et al. Moving the retina: choroidal modulation of refractive state. Vision Res. 1995;35:37-50. 
7. Wildsoet C, Wallman J. Choroidal and scleral mechanisms of compensation for spectacle lenses in chicks. Vision Res. 1995;35: 1175-1194.

8. Norton TT, Siegwart JT Jr. Animal models of emmetropization: matching axial length to the focal plane. J Am Optom Assoc. 1995;66:405-414.

9. Bartmann M, Schaeffel F. A simple mechanism for emmetropization without cues from accommodation or colour. Vision Res. 1994;34:873-876.

10. Smith EL III, Hung LF. Form-deprivation myopia in monkeys is a graded phenomenon. Vision Res. 2000;40:371-381.

11. Raasch TW. Spherocylindrical refractive errors and visual acuity. Optom Vis Sci. 1995;72:272-275.

12. Thibos LN, Wheeler W, Horner D. Power vectors: an application of Fourier analysis to the description and statistical analysis of refractive error. Optom Vis Sci. 1997;74:367-375.

13. Laskowski FH, Howland HC. Effect of experimentally simulated astigmatism on eye growth and refractive development in chicks [ARVO Abstract]. Invest Ophthalmol Vis Sci. 1996;37(3):S687. Abstract nr 3140.

14. Gwiazda J, Grice K, Held R, et al. Astigmatism and the development of myopia in children. Vision Res. 2000;40:1019-1026.

15. Irving EL, Callender MG, Sivak JG. Inducing ametropias in hatchling chicks by defocus: aperture effects and cylindrical lenses. Vision Res. 1995;35:1165-1174.

16. Smith EL Jr, Hung LF. Form-deprivation myopia in monkeys is a graded phenomenon. Vision Res. 2000;40:371-381.

17. Nickla D, Wildsoet C, Wallman J. Visual influences on diurnal rhythms in ocular length and choroidal thickness in chick eyes. Exp Eye Res. 1998;66:163-181.

18. Wallman J, Adams JI. Developmental aspects of experimental my- opia in chicks: susceptibility, recovery and relation to emmetropization. Vision Res. 1987;27:1139-1163.

19. Thibos LN, Cheng X, Phillips J, et al. Astigmatic deprivation of chicks produces myopia, but not astigmatism [ARVO Abstract]. Invest Ophthalmol Vis Sci. 2001;42(4):S58. Abstract nr 324.

20. Schmid K, Wildsoet CF. Natural and imposed astigmatism and their relation to emmetropization in the chick. Exp Eye Res. 1997;64: 837-847.

21. Schmid KL, Wildsoet CF. Effects on the compensatory responses to positive and negative lenses of intermittent lens wear and ciliary nerve-section in chicks. Vision Res. 1996;36:1023-1036.

22. Smith EL III, Huang J, Hung L-F. Cylindrical spectacle lenses alter emmetropization and produce astigmatism in young monkeys. In: Tokoro T, ed. Myopia Updates: Proceedings of the 6th International Conference on Myopia. Tokyo: Springer; 1998:336-343.

23. Kee C-S, Hung L-F, Qiao Y, et al. Does the axis of astigmatism influence the effects of astigmatism on emmetropization in rhesus monkeys [ARVO Abstract]? Invest Ophthalmol Vis Sci. 2000;41(4): S738. Abstract nr 3932.

24. Woods RL, Bradley A, Atchison DA. Monocular diplopia caused by ocular aberrations and hyperopic defocus. Vision Res. 1996;36: 3597-606.

25. Winawer J, Wallman J. Temporal constraints on lens compensation in chicks. Vision Res. 2002;42:2651-2668.

26. Winawer JA, Zhu X, Park T, et al. Is myopic blur more important than sharp vision for positive-lens compensation [ARVO Abstract]? Invest Ophthalmol Vis Sci. 2000;41(4):S136. Abstract nr 698.

27. Zhu X, Winawer JA, Choi JW, et al. The effect of defocusing lenses depends on the temporal integration characteristics of the emmetropization mechanism [abstract]. 2002 Annual Meeting Abstract and Program Planner accessed at www.arvo.org. Association for Research in Vision and Ophthalmology. Abstract 2929. 